\title{
CCD photometry of distant comets. III
}

\section{Ensemble properties of Jupiter-family comets}

\author{
S. C. Lowry ${ }^{\star}$, A. Fitzsimmons, and S. Collander-Brown
}

APS Division, Department of Pure and Applied Physics, The Queen's University of Belfast, Belfast BT7 1NN, UK

Received 22 January 2002 / Accepted 10 October 2002

\begin{abstract}
We describe the results of a ground-based observational "snapshot" study of Jupiter-family comets in the heliocentric range 2.29 AU $\leq R_{\mathrm{h}} \leq 5.72 \mathrm{AU}$. Results are presented based on observations from the $1 \mathrm{~m}$ JKT on the island of La Palma. A total of 25 comets were targeted with 15 being positively detected. Broad-band VRI photometry was performed to determine dimensions, colour indices, and dust production rates in terms of the "Af $\rho$ " formalism. The results for selected comets are compared with previous investigations. Ensemble properties of the Jupiter-family population have been investigated by combining the results presented here with those of Lowry et al. (1999), and Lowry \& Fitzsimmons (2001). We find that the cumulative size distribution of the Jupiter-family comets can be described by a power law of the form; $\Sigma(>r) \propto r^{-1.6 \pm 0.1}$. This size distribution is considerably shallower than that found for the observed Edgeworth-Kuiper belt objects, which may reflect either an intrinsic difference at small km-sizes in the belt, or the various processes affecting the nuclei of comets as their orbits evolve from the Edgeworth-Kuiper belt to the inner Solar system. Also, there would appear to be no correlation between nuclear absolute magnitude and perihelion distance. Finally, for the sample of active comets, there is a distinct correlation between absolute $R$ band magnitude and perihelion distance, which can be explained by either a discovery bias towards brighter comets or in terms of "rubble" mantle formation.
\end{abstract}

Key words. comets: general - techniques: photometric

\section{Introduction}

Knowledge of the size distribution of the various cometary groups is vital to constrain early solar system accretion models. Determining the size distribution of the Jupiter-family comets may be the only way to probe the size distribution of the smaller Edgeworth-Kuiper belt objects too faint to be seen with current instrumentation. Although this family of comets have generally spent some time in the inner solar system, some of this population are recent arrivals and thus represent the least altered samples from the Edgeworth-Kuiper belt.

Unfortunately cometary nuclei are extremely difficult to observe. One technique is to observe them at large heliocentric distances where the sublimation of surface volatiles is so low that any photometric measurements made are dominated by light reflected from the nucleus rather than from the dust coma. Indeed, nucleus size estimates have continued to decrease as more effective techniques emerge which are able to more accurately quantify the coma contamination. With the exception of the Centaurs and C/1995 O1 (Hale-Bopp), current

Send offprint requests to: S. Lowry, e-mail: Stephen.Lowry@jpl .nasa.gov

* Present address: Jet Propulsion Laboratory, Earth \& Planetary Sciences Division, 4800 Oak Grove Drive, Pasadena, CA 91109, USA. measurements of nuclear radii range from $0.35 \mathrm{~km}<r_{\mathrm{N}}<$ $15.40 \mathrm{~km}$ (Meech 2002 and references therein; Mueller 1992; Hainaut et al. 1998; Boehnhardt et al. 1999; Lamy et al. 1998; Lamy et al. 1999).

Few studies of distant comets have been carried out in the past. Meech \& Hainaut (1997) have an on-going long-term programme to obtain CCD images of short and long period comets over a wide range of heliocentric distances to compare activity levels and obtain information about primordial and evolutionary differences between comets with different dynamical histories. Other long-term ground based observational programs of distant comets currently underway include those of Licandro et al. (2000) and Fernández et al. (1999). Also, the Hubble Space Telescope has been used to obtain high spatial resolution CCD images of comets (Lamy \& Toth 1995), and has so far proved extremely effective. The largest study of cometary activity to date was the photoelectric photometry performed by A'Hearn et al. (1995), but in this study only $~ 3 \%$ of the observations were at a heliocentric distance $>3 \mathrm{AU}$.

We present here broad-band CCD photometry of distant Jupiter-family comets obtained using the $1 \mathrm{~m}$ Jacobus Kapteyn Telescope (JKT) in June 1999. These observations were obtained to supplement those discussed in Lowry et al. (1999), and Lowry and Fitzsimmons (2001) (hereafter referred to as Papers I and II respectively). The observations presented here 
and in Papers I and II are "snapshot" in nature. In other words, we are observing the comets at only one point in the rotational lightcurve. "Snapshot" observations have the disadvantage of sacrificing rotational information but have the advantage that a larger number of comets can be sampled at any given time.

Section 2 briefly describes the observations, the instrumental set-up, and the photometric calibration procedure. Section 3 outlines the analysis of each cometary observation and the results obtained. In order to be consistent with Papers I and II we have divided this section, with each subsection describing separately the results and analysis of comets that were undetected, unresolved, and active. In Sect. 4 we discuss in more detail the results obtained, as well as comparing our results with those of previous investigations. In Sect. 5 we have combined the results obtained here with those presented in Papers I and II in an attempt to describe the ensemble properties of the Jupiter-family comet population. A brief summary and some concluding remarks are provided in Sect. 6.

\section{Observations and photometric calibration}

$\mathrm{CCD}$ imaging of comets in the range $2.29 \mathrm{AU} \leq R_{\mathrm{h}} \leq 5.72 \mathrm{AU}$ was carried out on the nights of the 8th -15 th June 1999 using the $1 \mathrm{~m}$ Jacobus Kapteyn Telescope (JKT) on the Island of La Palma. Only the nights of the 8th, 11th, and 13th were photometric. A TEK $1024 \times 1024$ pixel CCD was used at the f/15 cassegrain focus resulting in an image scale of 0.33 arcsec per pixel. All images were taken through Harris $V, R$, and $I$ band filters, and all cometary images were obtained with the telescope tracking at the sidereal rate of motion. Exposure times of each frame depended on the apparent rate of motion of the object as projected onto the plane of the sky. Exposure times were adjusted so that the comet remained within the FWHM of the stellar-background PSF, which is $\sim 0.9^{\prime \prime}$ for the JKT under good conditions. This method prevented the comet smearing across the image, which in turn improved the $S / N$ of the target and greatly increased the possibility of detection. Additionally, a direct and more accurate comparison of the cometary profile with that of background stars was therefore possible. Exposure times ranged from 120 to $600 \mathrm{~s}$.

A log of all cometary observations, and the observational circumstances for each of the 25 targeted comets is listed in Table 1 . Out of the 25 targeted comets, 15 were positively detected and identified. Object detection was achieved by their known motion relative to the background stars. For the brighter targets, detection was immediate, but for others (particularly 19P/Borrelly and 118P/Shoemaker-Levy 4) several images were shifted and then coadded to reveal the comet by increasing the $S / N$. Additional search and detection criteria included astrometry of field stars to enable inspection of the exact predicted position of the comet.

The bias level was seen to fluctuate slightly throughout each night of observation, therefore the overscan region of each frame was utilized and each image was subsequently accurately bias-subtracted. Throughout the observing run a series of twilight sky exposures were obtained through each of the $V, R$, and $I$ band filters. The data on photometric nights was calibrated using the standard field SA 109 from Landolt (1992). For a given filter, the constants from the transformation equations are all consistent at the 1-2 $\sigma$ level for nights 1,4 , and 6 , highlighting the stability of the system during the observations.

Unfortunately, atmospheric dust and substantial cloud cover during nights $2,3,5,7$, and 8 prevented a direct photometric calibration via the use of standard star observations taken on these particular nights. However, it was possible to photometrically calibrate many of the cometary target observations obtained on nights 2 and 3 using relative photometry of background field stars. In other words, we re-observed the stellar fields at the cometary positions of nights 2 and 3 during the next available photometric night. The re-observed stellar fields can thus be calibrated in the normal way and compared with their non-photometric equivalent which contains the comet.

\section{Results and analysis}

\subsection{Undetected comets}

Out of the original 25 targeted comets, 10 were undetected. These comets are listed in Table 2. Note that comet $118 \mathrm{P} /$ Shoemaker-Levy 4 was not found at the first attempt on night 3 , but was subsequently detected on night 6 . Considering the photometric data first, all available exposures of each comet were first aligned with respect to the background stars, then shifted according to their known rates of motion, and then finally median combined to increase the $S / N$. As this proved unsuccessful, limiting magnitudes were then determined by introducing artificial stars to the median combined frames at each comet's expected position. For each comet, the artificial stars were constructed using the average PSF of several bright, well isolated, background stars. To calculate the average background PSF, the individual frames for a given comet were shifted and coadded with the background stars aligned. This therefore provides an optimal representation of the brightness profile of a point source comet had it been visible on the shifted, median combined frame. The magnitudes of these artificial stars were gradually increased until they were no longer detectable, by visual inspection, against the background noise. An accuracy of \pm 0.1 mag was attainable for the limiting magnitudes.

Comets 64P/Swift-Gehrels, 111P/Helin-Roman-Crockett, $113 \mathrm{P} /$ Spitaler, and 118P/Shoemaker-Levy 4 (Night 3), were imaged during non-photometric conditions. Artificial stars were added to the median combined non-photometric frames to obtain instrumental $R$ band limiting magnitudes. These instrumental limiting magnitudes were converted to real limiting magnitudes via the use of calibration fields taken on subsequent photometric nights. As no trailing occurred, no correction to the limiting magnitudes was necessary. The limiting magnitudes were then used to derive upper limits to their effective nuclear radii by substituting them for $m_{\mathrm{R}}$ in the following equation (Russell 1916):

$A_{\mathrm{R}} r_{\mathrm{N}}^{2}=\frac{2.25 \times 10^{22} R_{\mathrm{h}}^{2} \Delta^{2} 10^{0.4\left(m_{\odot}-m_{\mathrm{R}}\right)}}{10^{-0.4 \alpha \beta}}$

where $A_{\mathrm{R}}$ is the geometric albedo in the $R$ filter, $r_{\mathrm{N}}[\mathrm{m}]$ is the effective radius of the nucleus, $R_{\mathrm{h}}[\mathrm{AU}]$ and $\Delta[\mathrm{AU}]$ are the heliocentric and geocentric distances respectively, $\alpha$ and $\beta$ are 
Table 1. Log for all comets observed in June 1999. All images were obtained with the telescope tracking at the sidereal rate. The observing conditions are also specified. The non-photometric data were adequately calibrated using relative photometry of background field stars (see Sect. 1). $R_{\mathrm{h}}$ and $\Delta$ are the heliocentric and geocentric distances respectively. $\alpha$ is the phase angle.

\begin{tabular}{|c|c|c|c|c|c|c|c|c|}
\hline Comet & UT date $^{\star}$ & $R_{\mathrm{h}}[\mathrm{AU}]$ & $\Delta[\mathrm{AU}]$ & $\alpha$ [deg.] & $\begin{array}{l}\text { Observing } \\
\text { conditions }^{\dagger}\end{array}$ & Filter & Airmass & $\begin{array}{c}\text { Exposure } \\
\text { time }[\mathrm{s}]\end{array}$ \\
\hline 2P/Encke & 12.191 & $3.93^{I}$ & 4.01 & 14.65 & $\mathrm{P}$ & $2 \times R$ & 1.695 & 120 \\
\hline \multirow[t]{3}{*}{ 14P/Wolf } & 10.102 & $3.98^{I}$ & 3.06 & 7.18 & NP & $3 \times R$ & 1.199 & 180 \\
\hline & 10.099 & & & & NP & $V$ & 1.187 & 180 \\
\hline & 10.106 & & & & NP & $I$ & 1.211 & 180 \\
\hline \multirow[t]{2}{*}{ 19P/Borrelly (Night 4) } & 12.079 & $5.36^{I}$ & 4.36 & 1.98 & $\mathrm{P}$ & $5 \times R$ & 2.004 & 120 \\
\hline & 12.070 & & & & $\mathrm{P}$ & $2 \times V$ & 1.999 & 120 \\
\hline 19P/Borrelly (Night 6) & 14.078 & $5.36^{I}$ & 4.35 & 1.75 & $P$ & $5 \times R$ & 2.010 & 120 \\
\hline 30P/Reinmuth 1 & 13.163 & $5.65^{A}$ & 5.11 & 9.20 & $\mathrm{P}$ & $3 \times R$ & 1.590 & 300 \\
\hline 43P/Wolf-Harrington & 13.983 & $4.46^{\circ}$ & 3.66 & 8.95 & $\mathrm{P}$ & $2 \times R$ & 1.871 & 180 \\
\hline \multirow[t]{2}{*}{ 44P/Reinmuth 2} & 9.023 & $4.26^{I}$ & 3.76 & 12.72 & $P$ & $R$ & 3.250 & 600 \\
\hline & 9.042 & & & & $P$ & $2 \times R$ & 4.485 & 300 \\
\hline 45P/Honda-Mrkos-Pajdusakova & 11.984 & $5.14^{I}$ & 4.17 & 3.69 & $\mathrm{P}$ & $3 \times R$ & 1.474 & 120 \\
\hline 46P/Wirtanen & 12.033 & $5.02^{O}$ & 4.03 & 2.77 & $\mathrm{P}$ & $3 \times R$ & 1.487 & 120 \\
\hline \multirow[t]{3}{*}{ 47P/Ashbrook-Jackson } & 13.960 & $4.03^{I}$ & 3.23 & 9.97 & $P$ & $2 \times R$ & 1.827 & 180 \\
\hline & 13.961 & & & & $\mathrm{P}$ & $V$ & 1.826 & 180 \\
\hline & 13.968 & & & & $\mathrm{P}$ & $I$ & 1.866 & 180 \\
\hline \multirow[t]{3}{*}{ 49P/Arend-Rigaux } & 13.944 & $3.34^{O}$ & 2.78 & 16.01 & $\mathrm{P}$ & $3 \times R$ & 1.087 & 180 \\
\hline & 13.940 & & & & $\mathrm{P}$ & $V$ & 1.079 & 180 \\
\hline & 13.947 & & & & $\mathrm{P}$ & $I$ & 1.092 & 180 \\
\hline 61P/Shajn-Schaldach & 11.022 & $4.39^{I}$ & 3.40 & 3.14 & NP & $3 \times R$ & 1.360 & 120 \\
\hline 64P/Swift-Gehrels & 11.144 & $3.43^{I}$ & 2.49 & 8.08 & NP & $3 \times R$ & 1.799 & 180 \\
\hline 67P/Churyumov-Gerasimenko & 14.022 & $5.72^{A}$ & 4.77 & 3.89 & $\mathrm{P}$ & $2 \times R$ & 1.655 & 180 \\
\hline 69P/Taylor & 10.017 & $4.03^{O}$ & 3.18 & 8.90 & NP & $3 \times R$ & 1.147 & 180 \\
\hline 75P/Kohoutek & 9.126 & $4.37^{I}$ & 3.74 & 11.26 & $\mathrm{P}$ & $3 \times R$ & 1.596 & 600 \\
\hline 83P/Russell 1 & 14.113 & $3.01^{O}$ & 2.14 & 11.84 & $P$ & $10 \times R$ & 1.152 & 150 \\
\hline 97P/Metcalf-Brewington & 9.140 & $4.76^{I}$ & 4.11 & 10.11 & $\mathrm{P}$ & $3 \times R$ & 1.159 & 300 \\
\hline \multirow[t]{3}{*}{ 103P/Hartley 2} & 8.912 & $4.57^{O}$ & 4.24 & 12.52 & $P$ & $R$ & 1.431 & 300 \\
\hline & 8.931 & & & & $P$ & $2 \times R$ & 1.575 & 600 \\
\hline & 8.917 & & & & $\mathrm{P}$ & $V$ & 1.462 & 300 \\
\hline 104P/Kowal 2 & 13.922 & $3.94^{O}$ & 3.31 & 12.66 & $\mathrm{P}$ & $3 \times R$ & 1.540 & 240 \\
\hline 111P/Helin-Roman-Crockett & 9.969 & $4.35^{\circ}$ & 3.49 & 7.95 & NP & $3 \times R$ & 1.340 & 240 \\
\hline 113P/Spitaler & 10.179 & $4.22^{I}$ & 3.43 & 9.59 & NP & $3 \times R$ & 1.753 & 300 \\
\hline 118P/Shoemaker-Levy 4 (Night 3) & 11.005 & $4.71^{O}$ & 3.72 & 3.00 & NP & $3 \times R$ & 1.323 & 120 \\
\hline 118P/Shoemaker-Levy 4 (Night 6) & 14.051 & $4.71^{O}$ & 3.73 & 3.50 & $\mathrm{P}$ & $10 \times \mathrm{R}$ & 1.348 & 180 \\
\hline 121P/Shoemaker-Holt 2 & 11.036 & $5.03^{O}$ & 4.02 & 1.92 & NP & $3 \times R$ & 1.360 & 150 \\
\hline \multirow[t]{3}{*}{ 137P/Shoemaker-Levy 2} & 11.182 & $2.29^{I}$ & 2.03 & 15.17 & NP & $3 \times R$ & 1.351 & 300 \\
\hline & 11.177 & & & & NP & $V$ & 1.350 & 300 \\
\hline & 11.187 & & & & NP & $I$ & 1.351 & 300 \\
\hline P/1993 X1 (Kushida-Muramatsu) & 11.174 & $4.11^{I}$ & 3.74 & 13.85 & $\mathrm{P}$ & $2 \times R$ & 1.625 & 300 \\
\hline
\end{tabular}

$\star$ June 1999, † P - Photometric, NP - Non Photometric

$I$ - Inbound (Pre-perihelion), $O$ - Outbound (Post-perihelion), $A$ - At aphelion.

the phase angle and phase coefficient respectively. $m_{\odot}$ and $m_{\mathrm{R}}$ are the apparent $R$ magnitudes of the sun and comet respectively. Table 2 lists the $3 \sigma$ limiting magnitudes and corresponding effective nuclear radii $3 \sigma$ upper limits. As normal, empirically derived values for the albedo of 0.04 (see Fig. 4) and phase coefficient of $0.035 \pm 0.005$ (Sekanina 1976; Meech \& Jewitt 1987; Jewitt \& Meech 1987; Jewitt \& Meech 1988;
Jewitt \& Luu 1989) are assumed, and additional uncertainties introduced through these assumptions will be discussed later.

The effective radii upper limits for comets 44P/Reinmuth 2 and $118 \mathrm{P} /$ Shoemaker-Levy 4 are regarded as extremely reliable as both objects were subsequently detected on nights 2 and 6 respectively, and were both within a few arcseconds of their predicted positions. Although 44P/Reinmuth 2 was 
Table 2. Limiting $R$ band magnitudes, nuclear radius upper limits (based on assumed albedos of 0.04 and 0.02 ), and $A f \rho$ upper limits for the undetected comets. Note that comet $118 \mathrm{P} /$ Shoemaker-Levy 4 was subsequently detected three nights later under improved weather conditions.

\begin{tabular}{|c|c|c|c|c|}
\hline \multirow[t]{2}{*}{ Comet } & \multirow[t]{2}{*}{$m_{\mathrm{R}}{ }^{*}$} & \multicolumn{2}{|c|}{$r_{\mathrm{N}}^{\star}[\mathrm{km}]$} & \multirow[t]{2}{*}{$A f \rho^{\star}[\mathrm{cm}]$} \\
\hline & & $A_{\mathrm{R}}=0.04$ & $A_{\mathrm{R}}=0.02$ & \\
\hline 30P/Reinmuth 1 & 21.8 & $\leq 3.8$ & $\leq 5.3$ & $\leq 13.6$ \\
\hline 44P/Reinmuth 2 & 21.2 & $\leq 3.0$ & $\leq 4.2$ & $\leq 11.4$ \\
\hline 46P/Wirtanen & 21.7 & $\leq 2.6$ & $\leq 3.7$ & $\leq 10.0$ \\
\hline 64P/Swift-Gehrels & 20.7 & $\leq 1.9$ & $\leq 2.6$ & $\leq 4.9$ \\
\hline 67P/Churyumov-Gerasimenko & 22.1 & $\leq 2.9$ & $\leq 4.1$ & $\leq 17.1$ \\
\hline 75P/Kohoutek ${ }^{\diamond}$ & 22.6 & $\leq 1.5$ & $\leq 2.2$ & $\leq 3.6$ \\
\hline 83P/Russell $1^{\diamond}$ & 23.3 & $\leq 0.5$ & $\leq 0.6$ & $\leq 0.7$ \\
\hline 111P/Helin-Roman-Crockett & 22.3 & $\leq 1.5$ & $\leq 2.2$ & $\leq 4.9$ \\
\hline $113 \mathrm{P} /$ Spitaler & 21.6 & $\leq 2.0$ & $\leq 2.8$ & $\leq 9.3$ \\
\hline 118P/Shoemaker-Levy 4 (Night 3) & 21.4 & $\leq 2.6$ & $\leq 3.6$ & $\leq 12.8$ \\
\hline P/1993 X1 (Kushida-Muramatsu) & 22.0 & $\leq 2.0$ & $\leq 2.8$ & $\leq 5.6$ \\
\hline
\end{tabular}

$* 3 \sigma R$ band limiting magnitudes, $\star 3 \sigma$ upper limits, $A_{\mathrm{R}}$ is the assumed nuclear albedo, $r_{\mathrm{N}}$ is the nuclear radius, $\diamond$ The effective radii upper limits for comets $75 \mathrm{P} /$ Kohoutek and $83 \mathrm{P} /$ Russell 1 should be treated with caution until further astrometric observations have been performed (see Sect. 3.1).

re-imaged on night 2 , the data could not be photometrically calibrated and thus unsuitable for effective radius measurements and was excluded from the analysis.

Astrometric measurements of comet 113P/Spitaler were performed within 1.5 years after these observations were performed and it was found that any change in the orbital elements was negligible. Its orbit was therefore accurate during the observations and the derived effective radii upper limit can be regarded as firm. For comets 30P/Reinmuth 1, 46P/Wirtanen, 67P/ChuryumovGerasimenko, and 111P/Helin-Roman-Crockett, astrometric observations have been performed within 3 years prior to June 1999. It is highly unlikely that non gravitational forces would alter the predicted positions of these objects sufficiently enough to remove them from our field of view when one considers the size of the field of view that was available to us (i.e. $\left.5.58^{\prime} \times 5.58^{\prime}\right)$ and the short timescales involved. Hence, the derived effective radii upper limits for these comets may also be regarded as firm. The most recent astrometric observations of comet P/1993 X1 (Kushida-Muramatsu) were obtained in June 1995. The effect of non gravitational forces on its orbital motion cannot yet be ascertained and only future observations can confirm the predicted position used for these observations. However, like comets 30P, 46P, 67P, and 111P, it is unlikely that non-gravitational forces acting upon the nucleus between June 1995 and June 1999 will remove the object from our large field of view.

Only comets 64P/Swift-Gehrels, 75P/Kohoutek and $83 \mathrm{P} /$ Russell 1 show reason for concern. It had been at least 8 years since they were last observed astrometrically. The probability of nuclear splitting or spontaneous disintegration occurring is therefore much higher. The derived effective radii upper limits for these comets should be treated with caution until additional astrometric observations are obtained. It is also noted that recent nuclear splitting has not been reported for any of the undetected comets of this section.
Af $\rho^{1}$ upper limits were also calculated based on the derived limiting magnitudes and are listed in Table 2 along with the effective radii upper limits. For each comet, $\rho$ is taken to be the point where the background PSF is $\sim 2 \%$ of its peak intensity. For example, in the case of 30P/Reinmuth 2, the background PSF of the median combined frame drops to $\sim 2 \%$ of its peak intensity at $\sim 3.3^{\prime \prime}$ from the central peak. $3.3^{\prime \prime}$ is equivalent to $\sim 12240 \mathrm{~km}$ at the comet, hence $\rho$ is taken to be this distance in the $A f \rho$ calculation.

\subsection{Unresolved comets}

Figures 1a-1c show typical $R$ band CCD images of some of the brighter unresolved comets that were observed. Where appropriate, stars and cosmic rays were removed from the vicinity of the comets using various tasks within the IRAF software package. In order to search for signs of activity, the scaled brightness profiles of background stars were compared with those of the comets, and in every case the profiles were indistinguishable, i.e. the objects appeared as unresolvable point sources. Aperture photometry was performed on these objects to yield $R$ filter apparent magnitudes and corresponding effective radii for assumed red geometric albedos of 0.02 and 0.04 . These values are listed in Table 3 along with upper limits for $A f \rho$.

To ascertain the significance of any possible coma contribution to the $R$ band apparent magnitudes and hence the nuclear radius measurements, the following expression is applied (Jewitt \& Danielson 1984):

$m_{\text {coma }}=\Sigma(r)-2.5 \log \left(2 \pi r^{2}\right)$

where $m_{\text {coma }}$ is the integrated magnitude of the assumed steady state coma within a circular aperture of radius $r$ [arcsec] and $\Sigma(r)\left[\mathrm{mag} / \operatorname{arcsec}^{2}\right]$ is the measured brightness at $r$. Here $r$ is taken to be the radius of the photometric aperture used in the

${ }^{1}$ For a description of this quantity, see A'Hearn et al. (1989). 

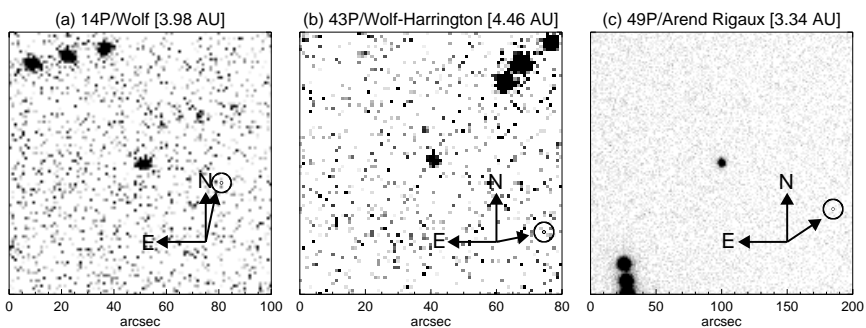

Fig. 1. $R$ band CCD images of selected unresolved comets. For each frame, the comets heliocentric distance and the direction of the sun are given. The size of each image is indicated by the arcsec scale along the $x$-axis. The total field of view is 5.63' squared.
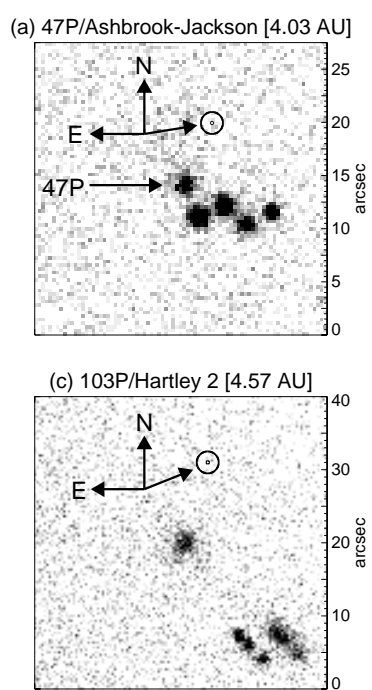

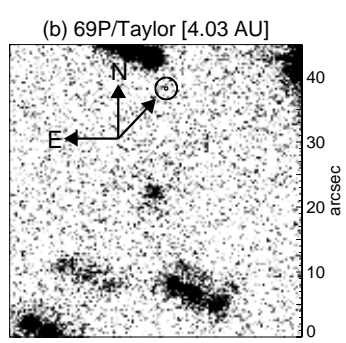

(d) $137 \mathrm{P} /$ Shoemaker-Levy 2 [2.83 AU]

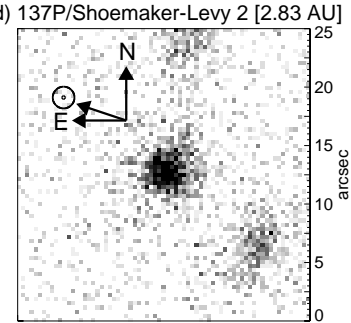

Fig. 2. $R$ band CCD images of the active comets before the removal of nearby stars. Note that only 47P/Ashbrook-Jackson and 103P/Hartley 2 were observed under photometric conditions. For each frame, the comets heliocentric distance and the direction of the sun are given. The size of each image is indicated by the arcsec scale along the $y$-axis. The total field of view is 5.63' squared.

determination of the comets real apparent magnitude. If we assume a minimum $S / N$ (per $\operatorname{arcsec}^{2}$ ) for detection of coma at $r$, we can calculate an lower limit to $\Sigma(r)$ and therefore a lower limit to $m_{\text {coma }}$. The derived $m_{\text {coma }}$ values are also listed in Table 3.

\subsection{Active comets}

Figures $2 \mathrm{a}-2 \mathrm{~d}$ show median combined $R$ band CCD images of the active comets. The observations were performed at airmasses $\leq 2$, and as with the comets of the previous section, nearby stars and cosmic rays were removed before any photometry was performed. For comets such as 103P/Hartley 2 and $137 \mathrm{P} /$ Shoemaker-Levy 2, cometary activity was easily recognized from their coma morphology. For 47P/AshbrookJackson and 69P/Taylor, a profile analysis was performed whereby the observed brightness profile of the comet was directly compared to that of nearby field stars.

For the active comets in this section, the value for $\rho$ used in the evaluation of the $A f \rho$ quantity is related to the aperture radius used for the total $R$ band magnitude measurement.
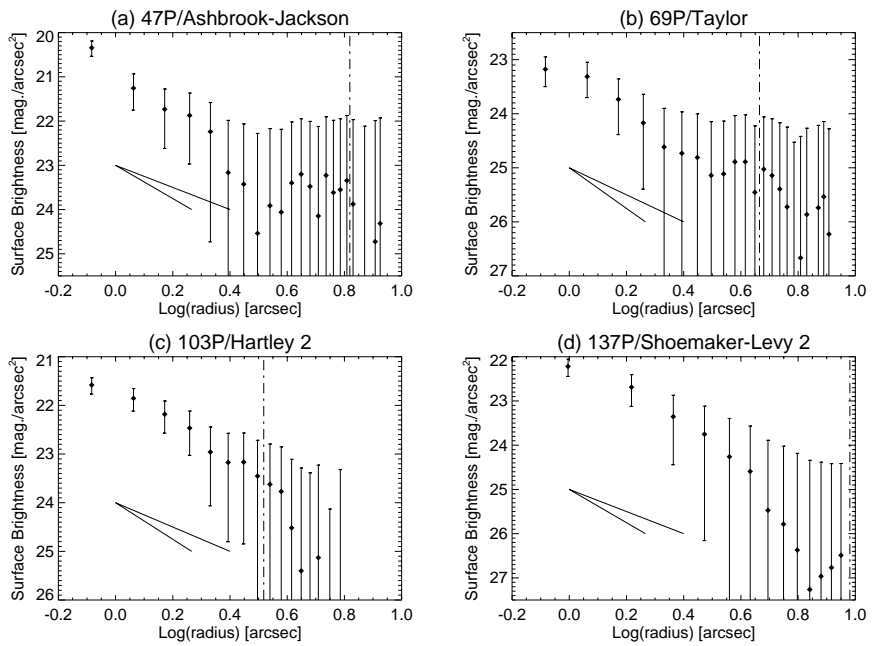

Fig. 3. Azimuthally averaged surface brightness profiles of the active comets. The two diagonal lines on each graph, with gradients of -1.5 and -1 , represent steady state coma models with and without the effects of radiation pressure respectively. The vertical dashed lines are the $A f \rho$ radii (i.e. the radii of photometric apertures used in the derived $A f \rho$ values).

In other words, a photometric radius of $6.6^{\prime \prime}$ is equivalent to $\sim 15500 \mathrm{~km}$ at the comet, therefore $\rho$ is taken to be this distance. Azimuthally averaged surface brightness profiles were determined for each of the four active comets and are shown in Fig. 3. In each case the cometary profiles are comparable to steady state coma models, within the experimental errors. Therefore the derived $A f \rho$ values listed in Table 3 are reliable.

$(V-R)$ and $(R-I)$ colour indices were measured and are listed in Table 4 . The total $R$ band magnitude was used to determine an upper limit to the effective nuclear radius. Also listed in Table 3, and labeled with the PSF superscript, are the $3 \sigma$ effective radius upper limits as determined using the scaled PSF method outlined in Sect. 3.3 of Paper I. This PSF method simply utilizes the stellar background PSF to reduce the effective radius upper limits of diffuse/active comets. Essentially, the stellar background PSF is scaled so that its peak brightness matches that of the comet, therefore the magnitude of the scaled stellar PSF is taken to be the maximum possible brightness of the nucleus at the time of the observations. Again, we assume typical values for the albedo of 0.02 and 0.04 to place upper limits on the nucleus sizes.

\section{Discussion}

\subsection{Nuclear radii measurements}

The effective nuclear radius measurements derived here range from $0.9 \mathrm{~km} \leq r_{\mathrm{N}} \leq 4.6 \mathrm{~km}$, and the upper limits derived span the range $0.5 \mathrm{~km} \leq r_{\mathrm{N}} \leq 7.5 \mathrm{~km}$ (for an assumed albedo of 0.04). These values are typical for Jupiter-family comets. The quoted errors for these values, as listed in Tables 2 and 3, are due to the photometry alone.

Following the discussion in Paper II, we have illustrated in Fig. 4 how the derived radius values vary as a function of the assumed albedo, for the unresolved comets. Filled circles 
Table 3. $R$ band magnitudes, nuclear radius measurements and upper limits (based on assumed albedos of 0.04 and 0.02 ), and $A f \rho$ values and upper limits for the detected comets. Note that the albedo values used for comets 49P/Arend-Rigaux and 2P/Encke are different as they have been previously measured.

\begin{tabular}{|c|c|c|c|c|c|c|}
\hline \multirow[t]{2}{*}{ Comet } & \multirow{2}{*}{$\begin{array}{c}R \text { band } \\
\text { magnitude }\end{array}$} & \multicolumn{2}{|c|}{$r_{N}[\mathrm{~km}]$} & \multirow[t]{2}{*}{$m_{\text {coma }}$} & \multirow{2}{*}{$\begin{array}{l}A f \rho \\
{[\mathrm{cm}]}\end{array}$} & \multirow{2}{*}{$\begin{array}{c}\text { Ap. radius }{ }^{\dagger} \\
\text { [arcsec] }\end{array}$} \\
\hline & & $A_{\mathrm{R}}=0.04$ & $A_{\mathrm{R}}=0.02$ & & & \\
\hline \multicolumn{7}{|l|}{ UNRESOLVED COMETS } \\
\hline 2P/Encke & $20.14 \pm 0.16$ & $4.43 \pm 0.32^{\circ}$ & $4.43 \pm 0.32^{\circ}$ & $19.06 \pm 0.03$ & $\leq 25.9^{\star}$ & 4.6 \\
\hline 14P/Wolf & $20.96 \pm 0.11$ & $2.33 \pm 0.12$ & $3.30 \pm 0.17$ & $19.90 \pm 0.05$ & $\leq 24.3^{\star}$ & 2.1 \\
\hline 19P/Borrelly (Night 4) & $22.63 \pm 0.36$ & $1.90 \pm 0.32$ & $2.69 \pm 0.45$ & $20.76 \pm 0.03$ & $\leq 20.9^{\star}$ & 2.1 \\
\hline 19P/Borrelly (Night 6) & $22.61 \pm 0.57$ & $1.91 \pm 0.51$ & $2.70 \pm 0.72$ & $18.92 \pm 0.03$ & $\leq 19.9^{\star}$ & 2.3 \\
\hline 43P/Wolf-Harrington & $20.81 \pm 0.14$ & $3.43 \pm 0.22$ & $4.86 \pm 0.32$ & $20.35 \pm 0.03$ & $\leq 32.0^{\star}$ & 2.3 \\
\hline 45P/H-M-P & $23.27 \pm 0.86$ & $1.34 \pm 0.55$ & $1.89 \pm 0.76$ & $18.47 \pm 0.03$ & $\leq 4.2^{\star}$ & 2.0 \\
\hline 49P/Arend-Rigaux & $19.51 \pm 0.05$ & $4.60 \pm 0.11^{\ddagger}$ & $4.60 \pm 0.11^{\ddagger}$ & $21.27 \pm 0.03$ & $\leq 23.8^{\star}$ & 3.3 \\
\hline 61P/Shajn-Schaldach & $23.27 \pm 0.56$ & $0.92 \pm 0.24$ & $1.31 \pm 0.34$ & $19.47 \pm 0.06$ & $\leq 9.8^{\star}$ & 2.0 \\
\hline 97P/Metcalf-Brewington & $22.23 \pm 0.40$ & $2.18 \pm 0.41$ & $3.09 \pm 0.57$ & $20.81 \pm 0.06$ & $\leq 9.8^{\star}$ & 3.3 \\
\hline 104P/Kowal 2 & $23.05 \pm 0.93$ & $1.04 \pm 0.46$ & $1.47 \pm 0.65$ & $20.14 \pm 0.03$ & $\leq 4.6^{\star}$ & 4.0 \\
\hline 118P/S-L 4 (Night 6) & $21.54 \pm 0.20$ & $2.42 \pm 0.22$ & $3.43 \pm 0.31$ & $20.23 \pm 0.03$ & $\leq 12.0^{\star}$ & 4.0 \\
\hline 121P/Shoemaker-Holt 2 & $22.66 \pm 0.76$ & $1.62 \pm 0.57$ & $2.29 \pm 0.82$ & $19.38 \pm 0.03$ & $\leq 16.7^{\star}$ & 2.6 \\
\hline \multicolumn{7}{|l|}{ ACTIVE COMETS } \\
\hline 47P/Ashbrook-Jackson & $19.07 \pm 0.15$ & $\leq 6.1^{P S F}$ & $\leq 8.6^{P S F}$ & - & $28.95 \pm 4.10$ & 6.6 \\
\hline 69P/Taylor & $19.93 \pm 0.10$ & $\leq 3.4^{P S F}$ & $\leq 4.8^{P S F}$ & - & $18.39 \pm 1.69$ & 4.6 \\
\hline 103P/Hartley 2 & $20.10 \pm 0.08$ & $\leq 5.8^{P S F}$ & $\leq 8.2^{P S F}$ & - & $37.83 \pm 2.75$ & 3.3 \\
\hline 137P/Shoemaker-Levy 2 & $18.71 \pm 0.14$ & $\leq 3.4^{P S F}$ & $\leq 4.8^{P S F}$ & - & $5.59 \pm 0.72$ & 9.6 \\
\hline
\end{tabular}

$\star 3 \sigma$ upper limits based on $R$ band magnitudes, $\dagger$ Radius of photometric aperture used to determine the $R$ band magnitudes and $A f \rho$ values, $A_{\mathrm{R}}$ is the assumed nuclear albedo, $r_{\mathrm{N}}$ is the nuclear radius, $\ddagger A_{\mathrm{R}}=0.03$ (Millis et al. 1988), $\diamond A_{\mathrm{R}}=0.05$ (Fernández et al. 2000), ${ }^{P S F}$ These upper limits were determined using the scaled PSF method outlined in Sect. 3.3 of Paper I.

Table 4. Additional $V$ and $I$ filter photometry for several unresolved and active comets. Their $R$ band magnitudes are reproduced to allow a direct comparison.

\begin{tabular}{lccccc}
\hline \hline Comet & $V$ & $R$ & $I$ & $(V-R)$ & $(R-I)$ \\
\hline UNRESOLVED COMETS & & & & & \\
14P/Wolf & $20.98 \pm 0.19$ & $20.96 \pm 0.11$ & $20.71 \pm 0.33$ & $0.02 \pm 0.22$ & $0.25 \pm 0.35$ \\
19P/Borrelly (Night 4) & $22.88 \pm 0.69$ & $22.63 \pm 0.36$ & - & $0.25 \pm 0.78$ & - \\
49P/Arend-Rigaux & $19.99 \pm 0.09$ & $19.51 \pm 0.05$ & $18.97 \pm 0.13$ & $0.49 \pm 0.11$ & $0.54 \pm 0.14$ \\
\hline ACTIVE COMETS & & & & & \\
47P/Ashbrook-Jackson & $19.42 \pm 0.17$ & $19.07 \pm 0.15$ & $18.88 \pm 0.27$ & $0.36 \pm 0.23$ & $0.19 \pm 0.31$ \\
103P/Hartley 2 & $20.41 \pm 0.09$ & $20.10 \pm 0.08$ & - & $0.32 \pm 0.12$ & - \\
137P/Shoemaker-Levy 2 & $19.24 \pm 0.25$ & $18.71 \pm 0.14$ & $18.26 \pm 0.30$ & $0.53 \pm 0.29$ & $0.45 \pm 0.33$ \\
\hline
\end{tabular}

The solar $(V-R)$ and $(R-I)$ colours are 0.36 and 0.28 respectively (Allen 1973; Fernie 1983).

highlight the position of each of the unresolved comets on their respective radius/albedo curves for the adopted albedos. Note that comets 2P/Encke and 49P/Arend-Rigaux have previously measured albedos of 0.05 (Fernández et al. 2000) and 0.03 (Millis et al. 1988) respectively.

Listed in Cols. 3 and 4 of Tables 2 and 3 are effective nuclear radii upper limits for the undetected and active comets respectively (for assumed albedos of 0.04 and 0.02). Upper limits based on the minimum measured albedo of 0.02 (A'Hearn et al. 1989) may be regarded as firm. Even if one applies a maximum axis ratio of 2.6 (Meech et al. 1993) and a minimum albedo of 0.02 to the undetected comets, their semi-major axes are all constrained to be below $8.7 \mathrm{~km}$. The uncertainty in the assumed phase coefficient of $\pm 0.005 \mathrm{mag} / \mathrm{degree}$ (as introduced in Sect. 3.1), will lead to an additional uncertainty of no greater than $0.2 \mathrm{~km}$ for the derived nuclear radii values and upper limits.

As with Papers I and II it is always appropriate to compare radius measurements and/or upper limits of those comets for which previous estimates exist. Such a comparison may allow additional constraints to be placed on nuclear axial ratios. With additional assumptions, this can lead to an estimate of the fractional active surface area present during previous apparitions. Table 5 lists those comets with previous nuclear radius measurements and upper limits derived from photometry, and lower limits derived from the amount of active area required to 

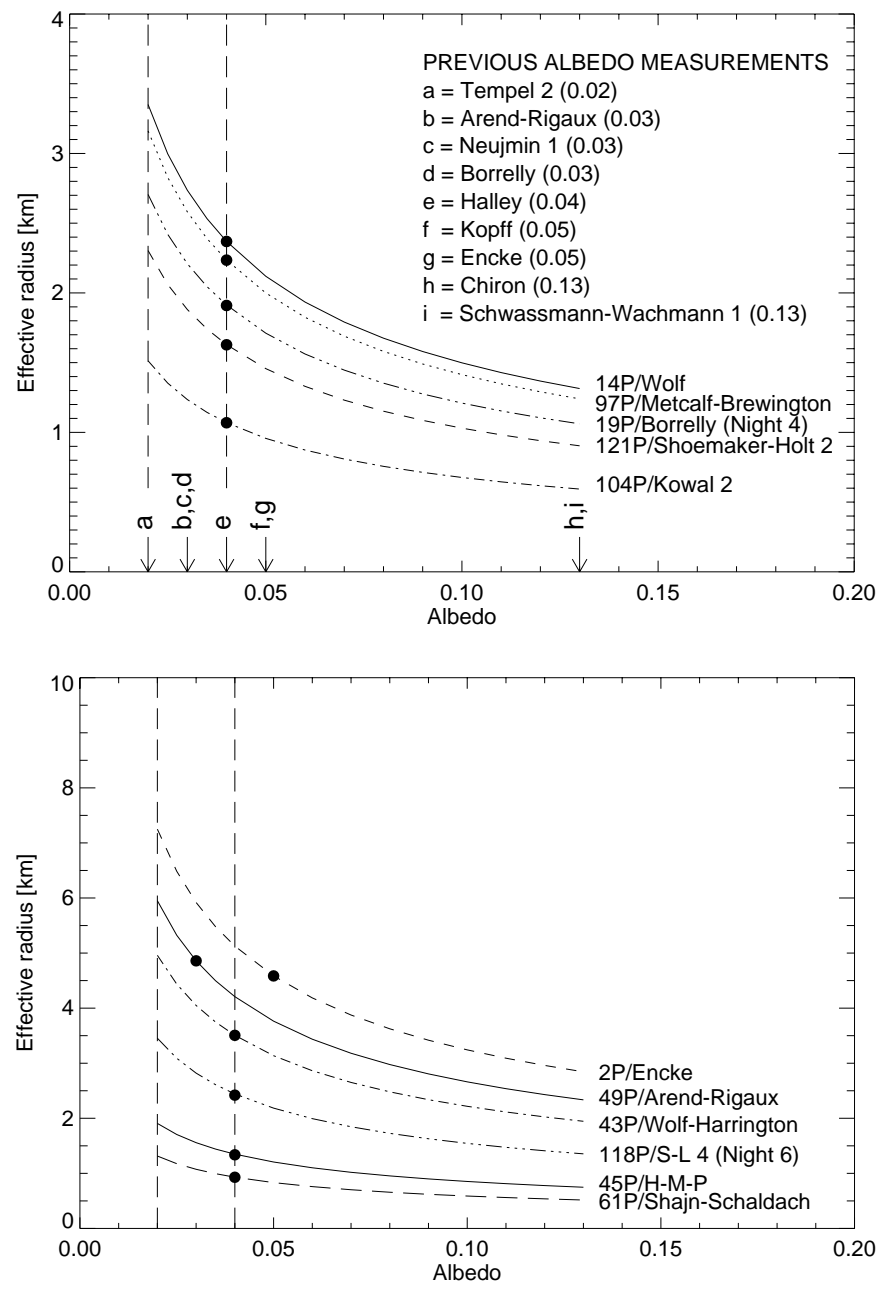

Fig. 4. Nuclear radius as a function of the assumed albedo for the unresolved comets of Sect. 3.2. The filled circles highlight the position of each of the unresolved comets on their respective radius/albedo curves for our adopted albedos. The region between the two vertical dashed lines represents the optimal range of assumed albedos, as the bulk of measured albedos reside within this region. The radius/albedo curves have been separated into two separate graphs for reasons of clarity. References: [a] A'Hearn et al. (1989), [b] Millis et al. (1988), [c] Campins et al. (1987), [d] Soderblom et al. (2002), [e] Keller et al. (1987), [f] Lamy et al. (2002), [g] Fernández et al. (2000), [h] Bus et al. (1989), [i] Cruikshank \& Brown (1983).

produce the measured $\mathrm{OH}$ production rates listed in A'Hearn et al. (1995).

Each of the $3 \sigma$ upper limits obtained from this work are consistent with the previous measurements, with the exception of 137P/Shoemaker-Levy 2. Licandro et al. (2000) observed this comet at a heliocentric distance of 4.24 AU (postperihelion). The comet appeared stellar on this occasion and had a mean absolute $V$ band magnitude of 14.5, which corresponds to a mean radius of $4.2 \mathrm{~km}$ for an assumed albedo of 0.04 . A variation of 0.4 magnitudes was observed, which corresponds to a projected axial ratio of 1.5 . Our $3 \sigma$ upper limit of $3.4 \mathrm{~km}$ (also assuming $A_{\mathrm{R}}=0.04$ ) is still significantly lower than this previous estimate even if one considers the lightcurve indicated by Licandro et al. If we assume a typical $(V-R)$ colour index of $0.50 \pm 0.10$ for the nucleus (Mueller 1992;
Jewitt \& Meech 1988; Boehnhardt et al. 1999; Licandro et al. 2000), then the absolute $R$ band magnitude $3 \sigma$ lower limit of 14.42 derived here increases $\Delta m$ to $0.61 \pm 0.10 \mathrm{mag}$, which in turn implies a new axial ratio lower limit of $1.8 \pm 0.2$. Considering that this comet had an observable coma during these observations, it is inevitable that this lower limit will increase further.

In contrast to the case of $137 \mathrm{P} /$ Shoemaker-Levy 2, the nuclear radii measurements for comets $45 \mathrm{P} /$ HondaMrkos-Pajdusakova and 97P/Metcalf-Brewington are somewhat larger than the previous measurements. Considering 45P/Honda-Mrkos-Pajdusakova first, Lamy et al. (1999) presents a mean radius value of $0.34 \mathrm{~km}$ based on a mean apparent $R$ band magnitude of 19.34 measured with the Hubble Space Telescope (HST). The apparent $R$ band magnitude ranged from approximately 19.30 to 19.43 throughout the two nights of observation. The corresponding absolute $R$ magnitude range is $\sim 19.98$ to 20.25 for an assumed phase coefficient of $0.035 \mathrm{mag} /$ degree (in order to be consistent with the measurements presented in this paper). The axial ratio for this comet must therefore be at least 1.3. Table 3 lists an apparent $R$ band magnitude of $23.27 \pm 0.86$, which corresponds to an absolute $R$ magnitude value of $16.48 \pm 0.86$. The absolute $R$ magnitude $3 \sigma$ lower limit is therefore 19.06, and after subtraction from the Lamy et al. value of 20.25, provides a new lower limit to $\Delta m$ of 1.19 , which in turn implies a new axial ratio lower limit of $\sim 3.0$. Some aspects of this result should be considered before it can be considered reliable. If this comet possessed an unresolved dust coma, then the nuclear absolute $R$ magnitude $3 \sigma$ lower limit would be greater than 19.06. This would then lower the $\Delta m$ value to yield a smaller axial ratio lower limit than the value presented here. However, it is reiterated that this comet was observed at a heliocentric distance of 5.14 AU where the sublimation of surface volatiles, particularly $\mathrm{H}_{2} \mathrm{O}$, is usually low. Also, Lamy et al. (1999) measured an extremely small Af $\rho$ value of $3.7 \mathrm{~cm}$ at a heliocentric distance of just $0.96-$ 0.97 AU. Furthermore, A'Hearn et al. (1995) measured a similar $A f \rho$ value of $3.5 \mathrm{~cm}$ when the comet was only $1.15 \mathrm{AU}$ from the sun (see Table 6). Hence it is highly probable that the dust production levels were negligible during our observations when the comet was over $5 \mathrm{AU}$ from the sun. This value of $\geq 3.0$, if confirmed, sets a new limit to the amount of elongation that can be expected for cometary nuclei. The largest previouslymeasured projected axial ratio was $\geq 2.6$ (Meech et al. 1993).

For 97P/Metcalf-Brewington, the radius value of $2.18 \pm$ $0.41 \mathrm{~km}$ derived here for an assumed albedo of 0.04 is significantly larger than the value of $1.4 \mathrm{~km}$ presented in Licandro et al. (2000) (also for an assumed albedo of 0.04). The comet appeared stellar and was at a post-perihelion heliocentric distance of 3.67 AU. A mean absolute $V$ band magnitude of 16.9 was measured, with a peak-to peak amplitude of 0.7 . A $\Delta m$ value of 0.7 implies an above average projected axial ratio of $\geq 1.91$. Based on this result, the possible range of effective radius values is therefore $1.18-1.63 \mathrm{~km}$. Our value of $2.18 \pm 0.41 \mathrm{~km}$ is actually consistent with this range at the $2 \sigma$ level.

49P/Arend-Rigaux was observed at an outbound heliocentric distance of $3.34 \mathrm{AU}$. These observations were performed 
just over five months after the December 1998 observations that were obtained using the $4.2 \mathrm{~m} \mathrm{WHT}$ (Paper II). It is apparent that the transition region from an outgassing state to one of complete inactivity may occur somewhere within the 2.1-3.3 AU zone. Of-course, heliocentric lightcurves of comets tend to be asymmetric, hence this finding only applies to the outbound leg of its orbit. The radius measurement derived here of $4.60 \pm 0.11$, for an assumed albedo of 0.03 (Millis et al. 1988), represents the bare nucleus value, and is completely consistent with previous values that span the range $3.8-6.8 \mathrm{~km}$ (see Paper II and references therein).

The case of comet 43P/Wolf-Harrington is interesting in the sense that each of the previous measurements are similar to the value derived here of $3.43 \pm 0.22 \mathrm{~km}$. The previous measurements are $3.3 \pm 0.7 \mathrm{~km}$ (Paper II), and $\leq 2.9 \mathrm{~km}$ (Licandro et al. 2000). Similar effective radii measurements and upper limits implies either the nucleus may be approximately spherical, or that the measurements were performed at similar points in the rotational lightcurve (excluding the Licandro et al. measurement). The only clue towards an elongated nucleus would be the fact that the Licandro et al. measurement was performed when the comet was highly active. The $2.9 \mathrm{~km}$ upper limit will undoubtedly be a significant overestimation of the effective radius, therefore the actual effective radius would not be consistent (at the $3 \sigma$ level) with our value.

Several of the targets from this particular observing run are possible or established targets of current and future spacecraft missions. Such targets include 2P/Encke, 19P/Borrelly, and $46 \mathrm{P} /$ Wirtanen. Comet $2 \mathrm{P} /$ Encke was one of the possible three or more targets of the recently failed Comet Nucleus Tour (CONTOUR). This comet has been studied extensively in the past with a range of nuclear radius values being published (see Fernández et al. 2000 and references therein). The effective radius value derived here of $4.43 \pm 0.32 \mathrm{~km}$ falls within the total range of previous values (i.e. $0.25-5.00 \mathrm{~km}$ ). As with 49P/Arend-Rigaux, this comet is one of the few to have its nuclear albedo measured. Using simultaneous visible and infrared observations, Fernández et al. (2000) measured an albedo of $0.05 \pm 0.02$ and an effective radius of $2.4 \pm 0.3 \mathrm{~km}$. If we consider the observed rotational lightcurve from Fernández et al. (2000) then we can argue that our photometry is consistent with their measurements. In other words, our observations may have taken place near the peak of the rotational lightcurve.

Comet 19P/Borrelly was observed previously with the HST in November 1994 (Lamy et al. 1998). By assuming an albedo of 0.04 and that the rotational axis is pointing in the direction determined by Sekanina (1979), the semi-axis were shown to be $4.40 \pm 0.30 \mathrm{~km}$ and $1.80 \pm 0.15 \mathrm{~km}$. A rotational period of $25.0 \pm 0.5$ hours was also found. Recently, this comet was the flyby target of the Deep Space 1 mission, the encounter occurring on September 22, 2001. Imaging by the spacecraft resolved the nucleus and revealed an average geometric albedo of $0.030 \pm 0.005$ (Soderblom et al. 2002). We observed this comet with the JKT on two separate nights. The observations were separated in time by 48 hours, and on both occasions an effective radius of $\sim 1.91 \mathrm{~km}$ was found. The comet was therefore observed on both nights near the same point in the rotational lightcurve (i.e. at the brightness minimum in this case). This acts as independent support for the above rotation period at the $2 \sigma$ confidence level. It is also apparent that the rotational state has remained virtually unchanged since the HST observations, despite prolonged outgassing during this period.

Comet 46P/Wirtanen is the target of the upcoming ROSETTA comet orbiter mission, scheduled for launch in January 2003. Previous observations show this cometary nucleus to be an extremely small object with a radius of $\sim 0.6$ $0.7 \mathrm{~km}$ (Lamy et al. 1998; Möhlmann 1999). An effective radius upper limit of $\leq 2.6 \mathrm{~km}$ was found, which is consistent with the previous work.

\subsection{Afo measurements}

Brightness profile analysis revealed coma activity for only 4 of the 25 targeted comets. The Af $\rho$ values obtained for these active comets range from $5.6 \mathrm{~cm} \leq A f \rho \leq 37.8 \mathrm{~cm}$, whereas the Af $\rho$ upper limits obtained for the undetected and unresolved comets range from $0.7 \mathrm{~cm} \leq A f \rho \leq 32.0 \mathrm{~cm}$. The complete range of $A f \rho$ values and upper limits observed on this occasion is rather small relative to that observed in Papers I and II. Poor seeing may explain why a large fraction of the detected comets appeared stellar, but from the observed range of $A f \rho$ values (which are a measure of the actual dust production levels) it would appear that the reduced levels of distant activity are real and not an artifact of instrumental or atmospheric limitations.

Table 6 compares the present $A f \rho$ values with those listed in Paper I, Paper II, and A'Hearn et al. (1995) derived at different heliocentric distances. Note that the A'Hearn et al. measurements were performed when the comets were at heliocentric distances $\leq 1.82 \mathrm{AU}$, whereas the previous measurements from Papers I and II were performed when the comets were at heliocentric distances $\geq 3.63 \mathrm{AU}$ (with the exception of comet 49P/Arend-Rigaux). For comets 19P, 43P, 46P, 49P, 67P, 97P, and $103 \mathrm{P}$ the present $A f \rho$ values or upper limits are very much less than the A'Hearn et al. measurements, which is expected when one considers the difference in heliocentric distance.

None of the comets listed in Table 6 are obvious candidates for continuous sublimation throughout their orbits, but it is strongly suspected that several are inactive during aphelion. Such comets include 19P, 44P, 45P, and 97P. This conclusion is based on the comets displaying either, a) similar $A f \rho$ values at widely different heliocentric distances, indicating an inert body with a constant scattering cross-section (i.e. comet 45P), b) small $A f \rho$ upper limits at large heliocentric distances (i.e. comet 44P), or c) small $A f \rho$ upper limits at large heliocentric distances coupled with a rapid rate of change of dust production with heliocentric distance (i.e. comets 19P and 97P). Each of these four comets are detectable at large heliocentric distances, and given that they are almost certainly inactive during aphelion, they are excellent candidates for photometric studies of nuclear properties. For comets 43P, 46P, 67P, 69P, and 103P, the level of activity during aphelion remains uncertain, although their $A f \rho$ values or upper limits remain small at large heliocentric distances. 
Table 5. Comparison of nuclear radii measurements with previous values.

\begin{tabular}{|c|c|c|c|}
\hline Comet & $r_{\mathrm{N}}[\mathrm{km}]$ & Previous values $^{\diamond}[\mathrm{km}]$ & Ref. \\
\hline \multirow[t]{2}{*}{ 2P/Encke } & $4.43 \pm 0.32$ & $0.25-5$ & 1 \\
\hline & & $\geq 0.24$ & 2 \\
\hline \multirow[t]{2}{*}{ 19P/Borrelly (Night 4) } & $1.90 \pm 0.32$ & $4.4 \times 1.8$ & 3 \\
\hline & & $\geq 0.73$ & 2 \\
\hline \multirow[t]{2}{*}{ 43P/Wolf-Harrington } & $3.43 \pm 0.22$ & $3.3 \pm 0.7, \leq 2.9$ & 4,5 \\
\hline & & $\geq 0.42$ & 2 \\
\hline 44P/Reinmuth 2 & $\leq 3.0^{\star}$ & $\leq 1.4^{\star}, \sim 1.63$ & 6,7 \\
\hline \multirow[t]{2}{*}{ 45P/H-M-P } & $1.34 \pm 0.55$ & 0.34 & 8 \\
\hline & & $\geq 0.11$ & 2 \\
\hline \multirow[t]{2}{*}{ 46P/Wirtanen } & $\leq 2.6^{\star}$ & $0.60 \pm 0.02,0.73 \pm 0.23$ & 9,10 \\
\hline & & $\geq 0.39$ & 2 \\
\hline 47P/Ashbrook-Jackson & $\leq 7.5^{\star}$ & 3.0 & 5 \\
\hline 49P/Arend-Rigaux & $4.60 \pm 0.11$ & $3.8-6.8$ & 11 \\
\hline 64P/Swift-Gehrels & $\leq 1.9^{\star}$ & 1.5 & 5 \\
\hline $67 \mathrm{P} / \mathrm{C}-\mathrm{G}$ & $\leq 2.9^{\star}$ & $2.4 \pm 0.1^{\dagger}$ & 12 \\
\hline \multirow[t]{2}{*}{ 69P/Taylor } & $\leq 4.6^{\star}$ & $3.6 \pm 0.7$ & 4 \\
\hline & & $\geq 0.25$ & 2 \\
\hline \multirow[t]{2}{*}{ 97P/Metcalf-Brewington } & $2.18 \pm 0.41$ & 1.4 & 5 \\
\hline & & $\geq 0.75$ & 2 \\
\hline \multirow[t]{2}{*}{ 103P/Hartley 2} & $\leq 6.7^{\star}$ & $\leq 5.0,0.56, \leq 5.9^{\star}$ & $5,13,14$ \\
\hline & & $\geq 0.62$ & 2 \\
\hline 137P/Shoemaker-Levy 2 & $\leq 3.4^{\star}$ & 4.2 & 5 \\
\hline
\end{tabular}

$r_{N}$ is the nuclear radius, $\diamond$ The lower limits are based on the OH production rates from A'Hearn et al. (1995) (see text), $\star 3 \sigma$ upper limits, $\dagger$ Based on a mean apparent $R$ band magnitude of $22.04 \pm 0.09$ (as presented in the respective reference) and an assumed albedo and phase coefficient of 0.04 and 0.035 respectively. References: [1] Fernández et al. (2000) and references therein, [2] A’Hearn et al. (1995), [3] Lamy et al. (1998b), [4] Paper I, [5] Licandro et al. (2000), [6] Paper II, [7] Lamy et al. (2000), [8] Lamy et al. (1999), [9] Lamy et al. (1998), [10] Möhlmann (1999), [11] See Paper II and references therein, [12] Mueller (1992), [13] Jorda et al. (2000), [14] Paper II.

\subsection{Colour indices}

Multi-filter photometry was performed on a total of 6 comets (see Table 4$)$. Where possible, both $(V-R)$ and $(R-I)$ colour indices were obtained, but for others such as $19 \mathrm{P} /$ Borrelly and $103 \mathrm{P} /$ Hartley 2 , only $V$ and $R$ band photometry was performed. The photometric errors associated with the bulk of these colour measurements are large, due to either low signal to noise and/or the limited accuracy that can be achieved through the photometric calibration of non-photometric data. Each of the active comets listed in Table 4 have colour indices consistent with the solar values at the $1 \sigma$ confidence level. The solar $(V-R)$ and $(R-I)$ colours, as transformed onto the photometric system used here, are 0.36 and 0.28 respectively (Allen 1973; Fernie $1983)$. For comet $14 \mathrm{P} /$ Wolf, the $(R-I)$ colour index is similar to the solar value, but the $(V-R)$ value is rather small, indicating a blue colour at shorter wavelengths. Such a small $(V-R)$ colour index could be explained by a lack of substantial mantle coverage, which acts to redden the surface.

In the case of comet 49P/Arend-Rigaux, good seeing, combined with relatively high signal to noise resulted in reduced error bars. The $(V-R)$ and $(R-I)$ colours are $0.49 \pm 0.11$ and $0.54 \pm 0.14$ respectively, which are significantly redder than the solar values. Given that this comet was certainly inactive on this occasion, it is clear that these colours represent the actual colours of the nuclear surface. In-phase optical and infrared rotational lightcurves have been observed for this comet (Millis et al. 1988), indicating a uniform surface composition. Hence, the $(V-R)$ and $(R-I)$ colour indices derived here should be unaffected by rotation. These colours are comparable to some TNOs (Green et al. 1997; Barucci et al. 2000), which are among the reddest known objects in the solar system. 49P/Arend-Rigaux has been studied extensively during past apparitions at visible and infrared wavelengths (see Paper II and references therein), and in each case the nucleus or dust coma was red in nature. Luu (1993) presents a nuclear spectrum of 49P/Arend-Rigaux at optical wavelengths and is also consistent with a "Type 2" rubble mantle. We therefore conclude that there has been no significant change in the nature of the mantle coverage since the previous investigations, despite recent periods of low activity (Paper II).

\section{Ensemble properties of Jupiter-family comets}

\subsection{Cumulative luminosity function}

Considering Papers I and II together with this work, the complete range of nuclear radii estimates for the unresolved comets is $0.92 \mathrm{~km}<r_{\mathrm{N}}<4.69 \mathrm{~km}$, while the range of $3 \sigma$ upper limits 
Table 6. Comparison of $A f \rho$ values with previous values listed in A'Hearn et al. (1995) [1], Paper I [2], Paper II [3].

\begin{tabular}{lccccc}
\hline \hline Comet & $A f \rho[\mathrm{cm}]$ & $R_{\mathrm{h}}[\mathrm{AU}]$ & $\begin{array}{c}\text { Previous } A f \rho \\
\text { value }[\mathrm{cm}]\end{array}$ & $R_{\mathrm{h}}[\mathrm{AU}]$ & Ref. \\
\hline 2P/Encke & $\leq 25.9^{\star}$ & $3.93^{I}$ & 28.84 & $0.86^{P}$ & 1 \\
19P/Borrelly & $\leq 20.9^{\star \diamond}$ & $5.36^{I}$ & 645.7 & $1.38^{P}$ & 1 \\
43P/W-H & $\leq 32.0^{\star}$ & $4.46^{O}$ & 134.9 & $1.82^{I}$ & 1 \\
& & & $\leq 28.4^{\star}$ & $4.87^{I}$ & 2 \\
44P/Reinmuth 2 & $\leq 11.4^{\star}$ & $4.26^{I}$ & $\leq 2.3^{\star}$ & $4.73^{I}$ & 3 \\
45P/H-M-P & $\leq 4.2^{\star}$ & $5.14^{I}$ & 3.5 & $1.15^{I}$ & 1 \\
46P/Wirtanen & $\leq 10.0^{\star}$ & $5.02^{O}$ & 112.2 & $1.12^{O}$ & 1 \\
49P/Arend-Rigaux & $\leq 23.8^{\star}$ & $3.34^{O}$ & 107.2 & $1.56^{O}$ & 1 \\
& & & $\leq 17.1^{\star}$ & $2.11^{O}$ & 3 \\
67P/C-G & $\leq 17.1^{\star}$ & $5.72^{A}$ & 208.9 & $1.38^{O}$ & 1 \\
69P/Taylor & $18.39 \pm 1.69$ & $4.03^{O}$ & $\leq 13.6^{\star}$ & $4.89^{I}$ & 2 \\
97P/M-B & $\leq 9.8^{\star}$ & $4.76^{I}$ & 275.4 & $1.61^{O}$ & 1 \\
103P/Hartley 2 & $37.83 \pm 2.75$ & $4.57^{O}$ & 245.5 & $1.04^{O}$ & 1 \\
& & & $49.3 \pm 4.8$ & $3.63^{O}$ & 3 \\
\hline
\end{tabular}

$I$ - Inbound (Pre-perihelion), $P$ - At perihelion, $O$ - Outbound (Post-perihelion), $A$ - At aphelion, $\star 3 \sigma$ upper limits, $\diamond$ Based on night 4 observations.

for the active and undetected comets is $0.5 \mathrm{~km}<r_{\mathrm{N}}<12.7 \mathrm{~km}$. An albedo of 0.04 was assumed throughout, with the exception of comets for which the albedo has been previously measured. Appendix A brings together each nuclear radius result from Papers I, II, and III into one table. This table also states whether the comet was active, unresolved, or undetected, and whether the comet was on its inbound or outbound leg of its orbit.

In deriving the absolute magnitude of the active comets the following expression is used:

$R_{(1,1,0)}=m_{\mathrm{R}}-2.5 n \log _{10}\left(R_{\mathrm{h}}\right)-5 \log _{10}(\Delta)-\beta \alpha$

where $R_{\mathrm{h}}[\mathrm{AU}]$ and $\Delta[\mathrm{AU}]$ are the heliocentric and geocentric distances respectively, and $m_{\mathrm{R}}$ is the measured apparent magnitude. As normal, $\beta \alpha$ accounts for the effects of phase darkening, where $\alpha$ [deg.] is the phase angle, and $\beta$ [magnitudes/degree] is the linear phase coefficient (assumed to be $0.035 \pm 0.005$ ). A linear phase term is valid when one considers the small phase angles of the active comets observed during this study.

For active comets, the constant $n$ is generally assumed to have a value of 4 . Essentially, this term accounts for variations of the total scattering cross-section with heliocentric distance, which is caused by changes in the dust production levels with heliocentric distance. For the unresolved and undetected comets, we assume $n=2$, as expected for inert bodies.

Figure 5a plots the cumulative number of comets with magnitudes brighter than $R_{(1,1,0)}$ versus $R_{(1,1,0)}$ for the active and unresolved comets combined, as-well as for the unresolved comets only. Considering the curve where the data for the active comets and unresolved comets are combined, it is clear that the slope of this curve is best represented by a broken power law for absolute magnitudes brighter than 15.5. Therefore the Cumulative Luminosity Function (CLF) of the absolute
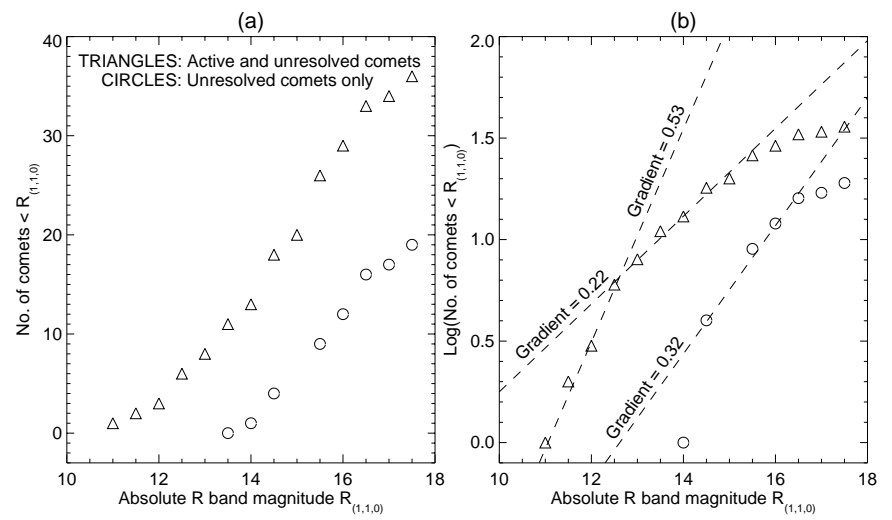

Fig. 5. (a) The number of comets $<R_{(1,1,0)}$ versus absolute $R$ band magnitude $R_{(1,1,0)}$ for the active and unresolved comets combined (triangles), as-well as the number of comets $<R_{(1,1,0)}$ versus $R_{(1,1,0)}$ for the unresolved comets only (circles). (b) Same as (a), but with a logarithmic $y$ axis. A slope of $0.32 \pm 0.02$ for the unresolved comets represents these data well, within the range $14.5<R_{(1,1,0)}<16.5$. These plots are derived from the amalgamation of results from Papers I and II, and this work (see Appendix A).

magnitudes of our entire sample of 33 detected comets can be described by:

$\log \left[\Sigma\left(<R_{(1,1,0)}\right)\right] \propto \begin{cases}(0.53 \pm 0.04) R_{(1,1,0)}, & R_{(1,1,0)} \leq 12.5 \\ (0.22 \pm 0.02) R_{(1,1,0)}, & 12.5<R_{(1,1,0)}<15.5\end{cases}$

where the gradients were found by applying a least squares fit to the data points within the given magnitude ranges (see Fig. 5b).

More important is the CLF of the unresolved comets. Fernández et al. (1999) have addressed this issue, but they used data from many different sources, with some dating back as 
far as 1950. The vast majority of their 'best estimates' for the nuclear magnitudes derive from inconsistent reduction methods and a significant portion rely upon less-than-optimal coma subtraction techniques. In addition, one cannot be sure if the derived magnitudes have been transformed onto a common photometric system. This makes inter-comparison of data between individual comets extremely difficult. Nevertheless, they have used these data in an attempt to constrain the CLF of the Jupiter-family comets. They plot $\log \left[\Sigma\left(<V_{(1,1,0)}\right)\right]$ versus absolute visual nuclear magnitude $V_{(1,1,0)}$ for comets with perihelion distances $<2 \mathrm{AU}$. Using these data, Fernández et al. find that the CLF follows a linear relation within the small magnitude range of $15.25<V_{(1,1,0)}<16$, with a gradient of 0.54 .

As shown in Fig. 5b, a least-squares fit to our data for unresolved nuclei implies a slope for the CLF of $0.32 \pm 0.02$, within the large absolute magnitude range of $14.5<R_{(1,1,0)}<16.5$. This gradient is significantly different from Fernández et al. (1999), but as our data is derived using homogeneous reduction methods and is entirely CCD based, we believe that this slope represents the most realistic estimate of the size distribution of Jupiter-family comets to date.

\subsection{Size distribution and comparison to other populations}

Now that we have an estimate of the CLF slope for the Jupiterfamily comets, we can derive the size and mass distributions of the Jupiter-family population. The CLF for Jupiter-family comets is of the form:

$\log \left[\Sigma\left(<R_{(1,1,0)}\right)\right] \propto \alpha R_{(1,1,0)}$

where $\alpha$ is the slope of the CLF. This implies $\Sigma\left(>r_{\mathrm{N}}\right) \propto$ $10^{\alpha R_{(1,1,0)}}$, and from Eq. (1) we have $r_{\mathrm{N}} \propto 10^{-0.2 R_{(1,1,0)}}$. If we approximate the size distribution of Jupiter-family comets by a power law, such that the number of nuclei with radii $>r_{\mathrm{N}}$ is:

$\Sigma(>r) \propto r^{1-q}, q>1$,

then $10^{\alpha} \equiv 10^{0.2(q-1)}$ i.e. $\alpha=0.2(q-1)$. Following on from Sect. 5.1, we therefore find:

$\Sigma(>r) \propto r^{-1.6 \pm 0.1}$.

It is important to compare the observed CLF of the Jupiterfamily comets with that of the Edgeworth-Kuiper belt, as this is the most probable source region of the majority of the Jupiterfamily population (Fernández 1980). Many investigations into the size distribution of Trans-Neptunian Objects (TNOs) have been performed. Typical values for the slope of the CLF are $0.52 \pm 0.02$ (Chiang \& Brown 1999), $0.58 \pm 0.05$ (Jewitt et al. 1998), $0.60_{-0.10}^{+0.12}$ (Trujillo et al. 2001), 0.69 (Gladman et al. 2001), and $0.76_{-0.11}^{+0.10}$ (Gladman et al. 1998). These values are much larger than our measurement of $0.32 \pm 0.02$ for Jupiterfamily comets, but are for significantly larger objects. The TNO size distributions are measured only for objects with supposed diameters $\geq 100 \mathrm{~km}$, while that of the cometary nuclei is for objects $\leq 10 \mathrm{~km}$. As of yet, there is no independent confirmation of the detection of cometary-nucleus sized bodies in the Kuiper belt, and so direct comparison of their size distributions is uncertain at best. Intriguingly however, Kenyon \& Windhorst (2001) have shown that $\alpha \leq 0.48$ for radii less than $\sim 1 \mathrm{~km}$ by using Olbers's paradox, thereby proving the size distribution to be shallower for smaller TNOs.

It is unlikely that the size distribution of the ejected TNOs would be preserved upon entering the inner solar system, due to the various processes acting upon the nucleus that would inevitably change their physical characteristics. Such processes include tidal disruption by the giant planets (Sekanina 1997), fragmentation due to intense solar heating (Delahodde et al. 2000; Filippenko \& Chornock 2000), and nuclear sublimation. Unfortunately, such processes would increase the slope of the CLF, and not decrease it as required by our data. Therefore how can we account for a CLF slope for the Jupiter-family comets of $0.32 \pm 0.02$ presented here? One explanation for this effect could be the observational bias towards the discovery of larger Jupiter-family comets, or at least those with a significant active surface area. If our measured value is truly intrinsic to the population however, then perhaps Solar heating leads to complete disintegration of small cometary nuclei or at least their rapid diminution to below observational detection limits, which would result in a further decrease in the slope of the CLF.

If indeed there is a progressive decrease in the slope of the CLF as comets evolve from the Edgeworth-Kuiper belt to the realm of the Jupiter-family comets, then a precise determination of the CLF of the Centaur population may prove valuable. Unfortunately, the discovery rate of Centaurs is relatively slow at present, but wide field CCD surveys are being conducted, with preliminary values for the slope of the Centaur CLF of $\sim 0.6$ (Sheppard et al. 2000) and 0.54 (Larsen et al. 2001).

Finally, it is interesting to note that our derived value for $\alpha$ of $0.32 \pm 0.02$ is similar to that for main-belt asteroids according to Jedicke \& Metcalf 1998, who found $\alpha \sim 0.3$ but with large variations. Also, recent estimates for the CLF slope parameter of Near Earth Objects are 0.35 (Rabinowitz 2000; Bottke et al. 2001), and 0.39 (Stuart 2001). Hence these two collisionally dominated populations display size distributions significantly different from the theoretically expected value of $\alpha=0.5$ (Dohnanyi 1969; Williams \& Wetherill 1994).

\subsection{Absolute magnitudes and orbital parameters}

Considering the activity levels of the Jupiter-family population as a whole, this survey clearly illustrates the diverse levels of activity present beyond $3 \mathrm{AU}$ from the Sun, and that for several comets the levels of activity are substantial. From Papers I, II, and III, the measured $A f \rho$ values for the active comets range from $5.6 \pm 0.7 \mathrm{~cm}$ to $299 \pm 11 \mathrm{~cm}$.

Correlations between the activity levels of the comets in our sample and their various orbital parameters were investigated. The only correlation we found for our sample was between intrinsic brightness and perihelion distance. Figure 6 plots the absolute $R$ band magnitude $R_{(1,1,0)}$ versus perihelion distance for every comet observed throughout the survey, and also includes most of the $3 \sigma$ upper limits obtained for the undetected comets. For the active comets in Fig. 6, the plotted magnitudes are the total magnitudes, i.e. nucleus plus coma. Several of the comets 


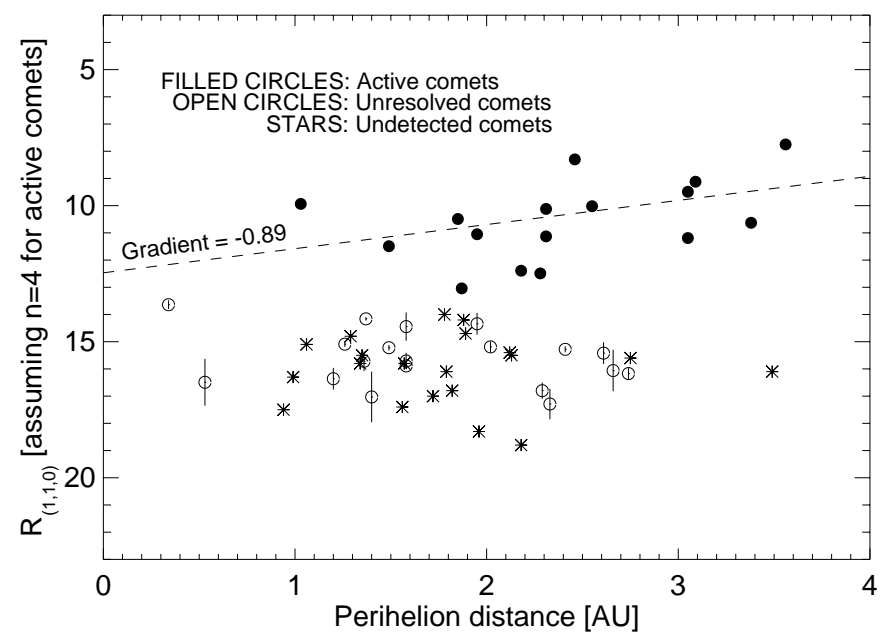

Fig. 6. This figure is a plot of the absolute $R$ band magnitude $R_{(1,1,0)}$ versus perihelion distance for the comets observed in this survey, and also includes most of the $3 \sigma$ upper limits obtained for the undetected comets. The active comets are represented by filled circles, whereas the unresolved and undetected comets are represented by open circles and stars respectively. For the active comets, there appears to be a distinct correlation between absolute total $R$ band magnitude and the comet's perihelion distance. A similar correlation between intrinsic brightness and perihelion distance is not seen for the unresolved and undetected comets, despite a wide range of perihelion distances (see Fig. 7).

in this sample were targeted on separate observing runs, therefore upper limits for undetected comets that were previously or subsequently detected, have been discarded. Also, for comets that were observed to be stellar in appearance on several occasions, the mean absolute magnitude is taken.

In Fig. 6, the active comets are represented by filled circles, whereas the unresolved and undetected comets are represented by open circles and stars respectively. For the active comets only, there appears to be a distinct correlation between absolute $R$ band magnitude and the comets perihelion distance, i.e. the intrinsic brightness increases with perihelion distance. Performing a least squares fit to these data points yields a slope of $-0.89 \pm 0.40$. Accurate knowledge of the parameter $n$ for each individual comet may reduce the scatter in the data points and hence the associated uncertainty, however it is believed that the upward trend seen in Fig. 6 for the active comets is a genuine feature.

This effect can be interpreted either as a discovery bias towards brighter comets, or in terms of mantle formation, specifically the "Rubble" mantle hypothesis. Recent arrivals to the inner Solar system should have relatively large fractional sublimating areas. As these new Jupiter-family comets are perturbed inwards, prolonged sublimation produces a rubble mantle which reduces the amount of free sublimating area and hence the brightness of the cometary coma at all heliocentric distances. The rubble mantle would continue to spread across the nuclear surface as the comet spends a larger fraction of its orbital period at progressively smaller heliocentric distances. This would imply the existence of a correlation between the

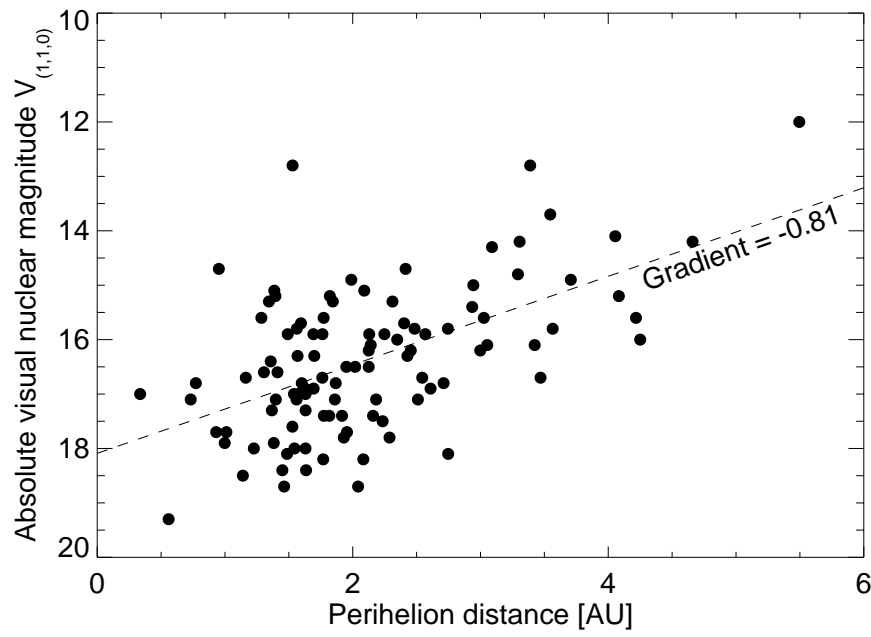

Fig. 7. This figure plots the "best estimates" for the visual nuclear magnitude $V_{(1,1,0)}$ versus perihelion distance. These data are from Fernández et al. (1999).

amount of active area and/or composition, with perihelion distance. Such a correlation between the amount of active area and perihelion distance has been seen previously by (A'Hearn et al. 1995), albeit extremely weakly.

A similar correlation between intrinsic brightness and perihelion distance is not seen for the unresolved and undetected comets, despite a wide range of perihelion distances. This finding is compared with the data presented in Fernández et al. (1999). Figure 7 plots their "best estimates" for the visual nuclear magnitudes versus perihelion distance. An apparent upward trend is seen and if one performs a least squares fit to the data points, then a linear relation with gradient $-0.81 \pm 0.12$ describes the data well. This value is remarkably similar to the slope seen for the active comets of Fig. 6. Hence, the illusion of an upward trend in Fig. 7 may be created if the comets with large perihelion distances are actually outgassing. Indeed, if one removes comets with perihelion distances beyond $3 \mathrm{AU}$ (which is only $16 \%$ of the comets in their sample), then one is left with a random scatter of data points, spread over a magnitude range similar to that of the unresolved and undetected comets of Fig. 6. Therefore, based on this argument and the data presented in Fig. 6, we conclude that there is, as of yet, no correlation between absolute nuclear magnitudes and perihelion distance.

\section{Summary}

CCD observations of comets in the region of $2.29 \mathrm{AU} \leq R_{\mathrm{h}} \leq$ 5.72 AU were carried out on the nights of the 8th -15 th June 1999 using the $1 \mathrm{~m} \mathrm{JKT}$ on the Island of La Palma. A total of 25 comets were targeted, from which photometric observations of 15 comets were obtained. Only four comets from this large sample displayed signs of distant activity. Those comets include 47P, 69P, 103P, and 137P. Based on a brightness profile analysis, eleven comets appeared stellar (2P, 14P, 19P, 43P, 45P, 49P, 61P, 97P, 104P, 118P, and 121P). The remaining 10 comets were undetected (30P, 44P, 46P, 64P, 67P, 75P, 83P, 111P, 113P, 
and $\mathrm{P} / 1993 \mathrm{X} 1$ ). VRI band photometry was performed on these comets to determine dimensions, colours, and dust production rates in terms of $A f \rho$.

The effective nuclear radius measurements for the unresolved comets range from $0.9 \mathrm{~km} \leq r_{\mathrm{N}} \leq 4.6 \mathrm{~km}$, and the upper limits for the active and undetected comets span the range $0.5 \mathrm{~km} \leq r_{\mathrm{N}} \leq 7.5 \mathrm{~km}$ (for an assumed albedo of 0.04). These values are typical for Jupiter-family comets. A range of assumed albedos are also discussed for the unresolved comets (see Fig. 4). For the active and undetected comets, firm $3 \sigma$ upper limits for an assumed albedo of 0.02 are also presented. Even if one applies a maximum axis ratio of 2.6 and a minimum albedo of 0.02 to the undetected comets, their semi-major axes are all constrained to lie below $8.7 \mathrm{~km}$.

The radius values derived here are compared with previous values. Those comets for which previous estimates exist are listed in Table 5. Each of the $3 \sigma$ upper limits obtained from this work are consistent with the previous measurements, with the exception of 137P/Shoemaker-Levy 2 . If the nucleus of $137 \mathrm{P} /$ Shoemaker-Levy 2 is modelled as a biaxial ellipsoid, then this inconsistency implies a bare nucleus axial ratio lower limit of $\geq 1.8 \pm 0.2$. Comparison of previous photometry of comet 45P/Honda-Mrkos-Pajdusakova with that presented here implies an exceptionally large axial ratio of 23.0. This value sets a new limit to the amount of elongation that can be expected for cometary nuclei. The possibility of an unresolved dust coma confounding this result is unlikely, as activity levels for this comet are intrinsically low, even for small heliocentric distances (A'Hearn et al. 1995; Lamy et al. 1999). Therefore, considering that this comet was observed beyond $5 \mathrm{AU}$ from the sun, dust contamination would be negligible and the above axial ratio lower limit may be regarded as firm. Comet 49P/Arend-Rigaux was observed at an outbound heliocentric distance of $3.34 \mathrm{AU}$, just over five months after the December 1998 observations. No jet structures were seen on this occasion, and it is apparent that, for the outbound leg of its orbit, the transition region from an outgassing state to one of complete inactivity may occur somewhere within 2.113.34 AU. Also, based on multiple observations, we present independent support of the nuclear rotation period of $\sim 25$ hours for comet 19P/Borrelly (Lamy et al. 1998b).

The $A f \rho$ values obtained for the active comets range from $5.6 \mathrm{~cm} \leq A f \rho \leq 37.8 \mathrm{~cm}$, whereas the $A f \rho$ upper limits obtained for the undetected and unresolved comets range from $0.7 \mathrm{~cm} \leq A f \rho \leq 32.0 \mathrm{~cm}$. These values were compared with previous values. There are no obvious candidates for continuous sublimation throughout their orbits, but it is strongly suspected that several are inactive around aphelion, namely comets 19P, 44P, 45P, 49P, and 97P.

Multi-filter photometry was performed on a total of 6 comets (14P, 19P, 49P, 47P, 103P, and 137P). A rather small $(V-R)$ colour index of $0.02 \pm 0.22$ for $14 \mathrm{P} /$ Wolf, could be explained by a lack of substantial mantle coverage. For 49P/Arend-Rigaux, the $(V-R)$ and $(R-I)$ colour indices are substantially redder than the solar values. This finding is consistent with previous investigations, which in turn implies that there has been no significant change in the nature of the mantle coverage since the previous investigations, despite recent periods of activity (Paper II).

By combining the results presented here with those from Papers I and II, ensemble properties of the Jupiter-family population were investigated. We find a value of $0.32 \pm 0.02$ for the slope of the Cumulative Luminosity Function. This value is similar to main-belt asteroids and Near-Earth Objects, but is shallower than that for Trans-Neptunian Objects. It is difficult to derive conclusions due to the different size ranges studied in these populations.

Correlations between the activity levels of the comets in our sample and their various orbital parameters were investigated. The only correlation to be found was that the extrapolated absolute $R$ band magnitude for the active comets was seen to decrease with increasing perihelion distance. This may be explained by a discovery bias towards brighter comets or may offer support to the hypothesis of rubble-mantle formation.

With regards to the physical properties of cometary nuclei, this survey has greatly improved the database of physical parameters of the Jupiter-family comet population. This homogeneously-reduced CCD data has allowed us to place vital constraints on the dimensions of many individual comets and on the size distribution of the Jupiter-family population as a whole. A substantial difference between the size distributions of TNOs and that of the Jupiter-family comets has been uncovered, which may reflect upon the various processes occurring on or within the nuclei of comets as their orbits evolve from the Edgeworth-Kuiper belt to the inner Solar system. Or, these differences may provide clues to the collisional processes and/or accretion mechanisms occurring within the protoplanetary disc. Also, there would appear to be no correlation between nuclear absolute magnitude and perihelion distance, as previously suggested.

This survey also demonstrates that Jupiter-family comets show diverse and, in many cases, substantial levels of activity beyond heliocentric distances of $3 \mathrm{AU}$, where the sublimation of $\mathrm{H}_{2} \mathrm{O}$ from the surface of the nucleus approaches negligible levels. Furthermore, comets such as 74P/Smirnova-Chernykh, $65 \mathrm{P} /$ Gunn, and $\mathrm{P} /$ Helin-Lawrence, are almost certainly continuously active throughout their entire orbits. Comets that display such high levels of distant activity render them excellent candidates for studies of the processes that induce distant sublimation.

Acknowledgements. We thank Dr. Paul Weissman of NASA's Jet Propulsion Laboratory and the anonymous referee for their detailed comments on earlier drafts of this paper. This work was carried out at The Queens University of Belfast with the support of the Department of Education for Northern Ireland. The Jacobus Kapteyn Telescope is operated on the island of La Palma by the Royal Greenwich Observatory at the Spanish Observatorio del Roque de los Muchachos of the Instituto de Astrofisica de Canarias. Image processing and photometry in this paper has been performed using the IRAF program. IRAF is distributed by the National Optical Astronomy Observatories, which is operated by the Association of Universities for Research in Astronomy, Inc. (AURA) under cooperative agreement with the National Science Foundation. 


\section{Appendix A: Absolute $R$-band magnitudes}

Table A.1. Absolute $R$-band magnitudes and corresponding effective radii for each of the targeted comets discussed in Papers I, II, and III. Also listed are each comet's heliocentric and perihelion distances. The appearance of the comet and whether it was on the inbound or outbound leg of its orbit, is also stated. Note that the upper/lower limits are all $3 \sigma$ limits.

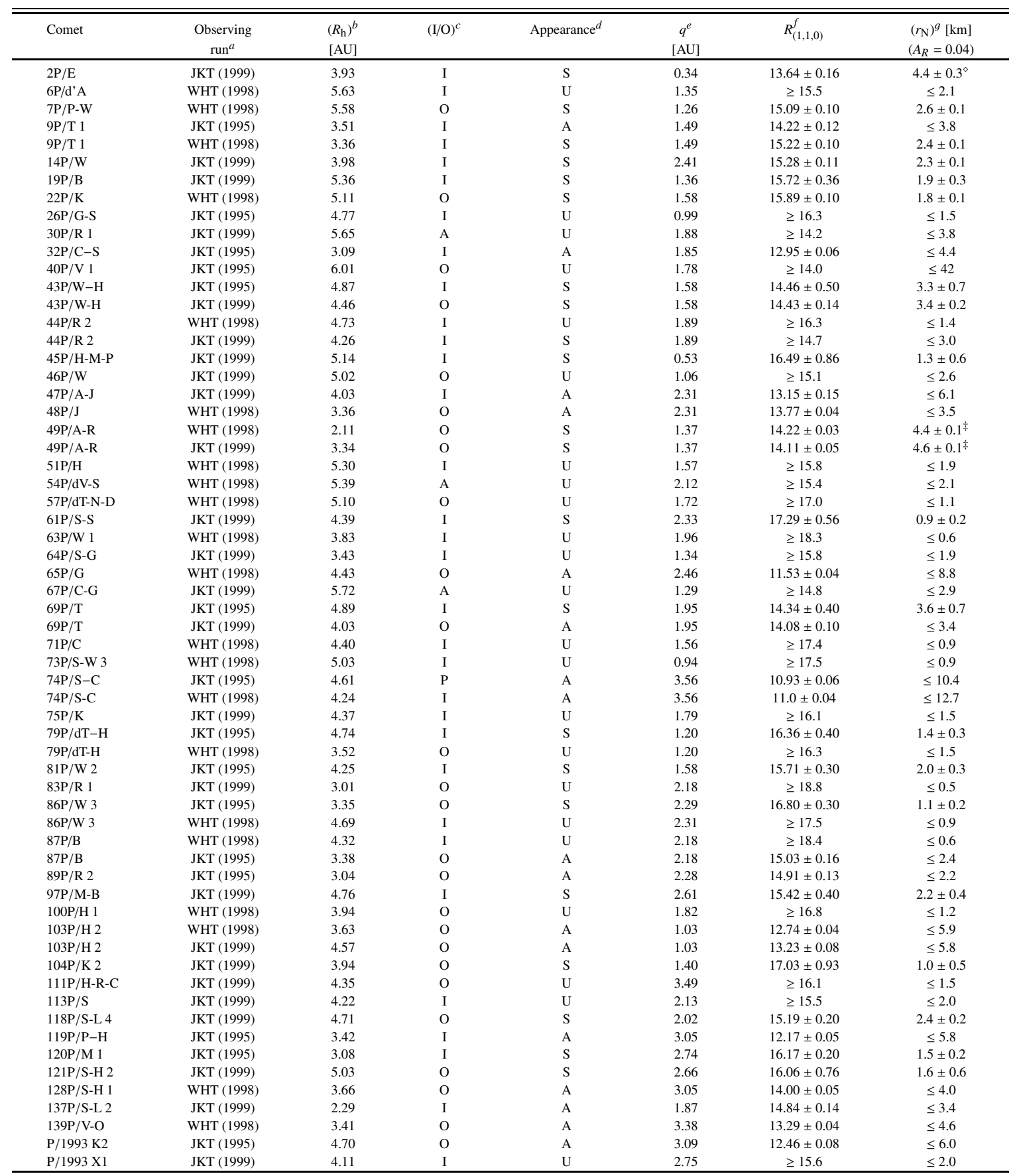

$a-$ JKT (1995) [Paper I], WHT (1998) [Paper II], JKT (1999) [Paper III].

$b-R_{\mathrm{h}}[\mathrm{AU}]$ is the heliocentric distance at the time of the observations.

$c$ - I - Inbound, O - Outbound, P - At perihelion, A - At aphelion.

$d$ - A - Active, S - Stellar, U - Undetected.

$e-q[\mathrm{AU}]$ is the perihelion distance.

$f$ - Absolute $R$-band magnitude (here, $n=2$ for the active comets, see Eq. (3)).

$g-r_{\mathrm{N}}[\mathrm{km}]$ is the effective radius based on $R_{(1,1,0)}\left(A_{\mathrm{R}}\right.$ is the assumed albedo).

$\diamond A_{\mathrm{R}}=0.05$ (Fernández et al. 2000).

$\ddagger A_{\mathrm{R}}=0.03$ (Millis et al. 1988). 


\section{References}

A’Hearn, M. F., Campins, H., Schleicher, D. G., \& Millis, R. L., 1989, ApJ, 347, 1155

A'Hearn, M. F., Millis, R. L., Schleicher, D. G., Osip, D. J., \& Birch, P. V. 1995, Icarus, 118, 223

Allen, C. W. 1973, in Astrophysical Quantities, 3rd Ed. (Athlone Press, University of London, London), 162

Barucci, M. A., Romon, J., \& Doressoundiram, A. 2000, AJ, 120, 496

Boehnhardt, H., Rainer, N., Birkle, K., \& Schwelm, G. 1999, A\&A, 341,912

Bottke, W. F., Morbidelli, A., Jedicke, R., et al. 2001, Icarus, in-press

Buratti, B. J., Soderblom, L. A., Britt, D. T., et al. 2001, BAAS, 33, 1091

Bus, S. J., Bowell, E., Harris, A. W., \& Hewitt, J. V. 1989, Icarus, 77, 223

Campins, H., A'Hearn, M. F., \& McFadden, L. 1987, AJ, 316, 847

Chiang, E. I., \& Brown, M. E. 1999, AJ, 118, 1411

Cruikshank, D. P., \& Brown, R. H. 1983, Icarus, 56, 377

Delahodde, C. E., Hainaut, O. R., \& Nakano, S. 2000, IAUC, 7467

Dohnanyi, J. S. 1969, JGR, 74, 2531

Fernández, J. A. 1980, MNRAS, 192, 481

Fernández, Y. R., Lisse, C. M., Käufl, H. U.,et al. 2000, Icarus, 147, 145

Fernández, J. A., Tancredi, G., Rickman, H., \& Licandro, J. 1999, A\&A, 337, 327

Fernie, J. D. 1983, PASP, 95, 782

Filippenko, A. V., \& Chornock, R. 2000, IAUC, 7468

Gladman, B., Kavelaars, J. J., Nicholson, P. D., Loredo, T. J., \& Burns, J. A. 1998, AJ, 116, 2042

Gladman, B., Kavelaars, J. J., Petit, J.-M., Morbidelli, A., \& Loredo, T. 2001, AJ, 122, 1051

Green, S. F., McBride, N., Ó Ceallaigh, Fitzsimmons, A., \& Williams, I. P. 1997, MNRAS, 290, 186

Hainaut, O. R., Meech, K. J., Boehnhardt, H., \& West, R. M. 1998, A\&A, 333, 746

Jedicke, R., \& Metcalfe, T. S. 1998, Icarus, 131, 245

Jewitt, D. C., \& Danielson, G. E. 1984, Icarus, 60, 435

Jewitt, D. C., \& Luu, J. 1989, AJ, 97, 1766

Jewitt, D. C., Luu, J. X., \& Trujillo, C. 1998, AJ, 115, 2125

Jewitt, D. C., \& Meech, K. J. 1987, AJ, 93, 1542

Jewitt, D. C., \& Meech, K. J. 1988, ApJ, 328, 974

Jorda, L., Lamy, P., Groussin, O., Toth, I., A'Hearn, M. F., \& Peschke, S. 2000, in ISO Beyond Point Sources: Studies of Extended Infrared Emission. ESA-SP 455, ed. R.J. Laureijs, K. Leech \& M.F. Kessler, 61
Keller, H. U., Delamere, W. A., Huebner, W. F., et al. 1987, A\&A, 187,807

Kenyon, S. J, \& Windhorst, R. A. 2001, ApJL, 547, 69

Lamy, P., \& Toth, I. 1995, A\&A, 293, L43

Lamy, P. L., Toth, I., A'Hearn, M. F., \& Weaver, H. A. 1999, Icarus, 140,424

Lamy, P. L., Toth, I., Jorda, L., et al. 2002, Icarus, 156, 442

Lamy, P. L., Toth, I., Weaver, H. A., et al. 2000, DPS meeting, 32, 36.04

Lamy, P. L., Toth, I., \& Weaver, H. A. 1998, A\&A, 337, 945

Lamy, P. L., Toth, I., Jorda, L., Weaver, H. A., \& A'Hearn, M. F. 1998b, A\&A, 335, L25

Larsen, J. A., Gleason, A. E., Danzl, N. M., et al. 2001, AJ, 121, 562

Landolt, A. U. 1992, AJ, 104, 340

Licandro, J., Tancredi, G., Lindgren, M., Rickman, H., \& Hutton, R. G. 2000, Icarus, 147, 161

Lowry, S. C., Fitzsimmons, A., Cartwright, I. M., \& Williams, I. P. 1999, A\&A, 349, 649

Lowry, S. C., \& Fitzsimmons, A. 2001, A\&A, 365, 204

Luu, J. X. 1993, Icarus, 104, 138

Meech, K. J. 2002, in Asteroids, Comets, Meteors 1996, COSPAR Coll., 10, in-press

Meech, K. J., Belton, M. J. S., Mueller, B., Dicksion, M., \& Li, H. 1993, AJ, 106, 1222

Meech, K. J., \& Hainaut, O. R. 1997, IAU Joint Discussion 6 on Interactions between Planets and Small Bodies

Meech, K. J., \& Jewitt, D. C. 1987, A\&A, 187, 585

Millis, R. L., A’Hearn, M. F., \& Campins, H. 1988, ApJ, 324, 1194

Möhlmann, D. 1999, Planetary and Space Science, 47, 971

Mueller, B. E. A. 1992, in Asteroids, Comets, Meteors 1991, (Harris, A., Bowell, E., Eds), 425

Rabinowitz, D., Helin, E., Lawrence, K., \& Pravdo, S. 2000, Nature, 403, 165

Russell, H. N. 1916, ApJ, 43, 173

Sekanina, Z. 1976, in The Study of Comets, Part 2, ed. B. Donn, M. Mumma, W. Jackson, M. A’Hearn, \& R. Harrington, 537

Sekanina, Z. 1979, Icarus, 37, 420

Sekanina, Z. 1997, A\&A, 318, L5

Sheppard, S. S., Jewitt, D. C., Trujillo, C. A., Brown, M. J. I., Ashley, M. C. B. 2000 , AJ, 120, 2687

Soderblom, A., Becker, T. L., Bennett, G., et al. 2002, Science, 296, 1087

Stuart, J. P. 2001, Science, 294, 1691

Trujillo, C. A., Luu, J. X., Bosh, A. S., \& Elliot, J. L. AJ, 122, 2740

Williams, D. R., \& Wetherill, G. W. 1994, Icarus, 107, 117 